\title{
Philonsorbonne
}

$3 \mid 2009$

Année 2008-2009

\section{Le pendule, la licorne et la figure. Comment les modèles scientifiques représentent les phénomènes}

\section{Marion VORMS}

\section{OpenEdition}

Journals

Édition électronique

URL : https://journals.openedition.org/philonsorbonne/237

DOI : 10.4000/philonsorbonne.237

ISSN : 2270-7336

Éditeur

Publications de la Sorbonne

Édition imprimée

Date de publication : 15 avril 2009

Pagination : 75-95

ISBN : 978-2-85944-624-6

ISSN : 1255-183X

Référence électronique

Marion VORMS, « Le pendule, la licorne et la figure. Comment les modèles scientifiques représentent les phénomènes », Philonsorbonne [En ligne], 3 | 2009, mis en ligne le 30 janvier 2013, consulté le 09 juin 2021. URL : http://journals.openedition.org/philonsorbonne/237 ; DOI : https://doi.org/10.4000/ philonsorbonne.237 


\title{
Le pendule, la licorne et la figure. Comment les modèles scientifiques représentent les phénomènes
}

\author{
Marion Vorms ${ }^{1}$
}

\section{Introduction}

Les modèles scientifiques sont principalement utilisés pour faire des prédictions et fournir des explications des phénomènes observables. On considère généralement que cette fonction prédictive et explicative provient du fait que les modèles sont des représentations des phénomènes: un modèle est un instrument qui tient lieu du système que l'on étudie ${ }^{2}$ en vertu d'une relation de représentation entre certaines de ses propriétés et certaines des propriétés du système. Cette relation de représentation permet aux scientifiques de faire des inférences et d'obtenir des informations à propos du système qu'ils étudient en raisonnant avec - et parfois littéralement en manipulant - le modèle.

Cette relation de représentation entre modèle et système physique est toutefois loin d'être clairement comprise et décrite par les philosophes des sciences : en vertu de quelles propriétés un modèle représente-t-il un système de manière à permettre au scientifique de faire des inférences utiles à la prédiction et à l'explication du comportement de ce système ?

Une réponse naturelle consiste à dire qu'un modèle scientifique représente son objet en vertu de son identité de structure - son isomorphisme - avec lui. Une telle conception s'applique particulièrement bien au cas des

1. Je remercie Anouk Barberousse, Julien Boyer, Steeves Demazeux, Roman Frigg, Jean Gayon, Paul Humphreys, Philippe Huneman, Cyrille Imbert, Philippe Lusson, ainsi que le relecteur anonyme de cette revue pour leurs commentaires sur des versions précédentes de cet article.

2. Je parlerai indifféremment de «système physique » ou «système cible » pour désigner la portion du monde extérieur dont il s'agit d'expliquer et de prédire le comportement. 
sciences mathématisées comme la physique, où les modèles sont identifiés à des structures mathématiques décrites par des équations. La conception dite «sémantique » des théories scientifiques ${ }^{3}$, défendue - pour ne citer que les noms les plus célèbres - par Patrick Suppes, Frederick Suppe, Bas Van Fraassen et Ronald Giere ${ }^{4}$, propose ainsi de définir une théorie comme la classe des modèles mathématiques ${ }^{5}$ qui satisfont les axiomes de cette théorie. Ces modèles sont isomorphes ${ }^{6}$ aux systèmes étudiés ${ }^{7}$. Par conséquent, la notion de représentation, dans ce cadre, ne pose pas de problème particulier : un modèle est une représentation en vertu de son isomorphisme au système étudié, ou, plus précisément, c'est parce que le modèle est isomorphe à ce système que l'on peut parler de représentation.

Cependant, il s'agit là d'une approche des théories scientifiques qui cherche à en dégager la structure logique, indépendamment de la manière dont ces théories sont apprises, développées et appliquées ${ }^{8}$. La question de la représentation est donc réduite au problème - non moins important - de l'application des mathématiques aux phénomènes observables, c'est-à-dire de la possibilité de décrire les phénomènes du monde extérieur par le biais de structures mathématiques. Résoudre cette question - ce que je

3. Cette conception se présente comme une critique de la conception dite «syntaxique » des théories scientifiques, issue des travaux des positivistes logiques, qui définit les théories comme des systèmes axiomatiques exprimés par des énoncés en logique du premier ordre, joints à leurs règles d'interprétation. La conception sémantique s'en démarque en considérant les théories comme des entités extra-linguistiques.

4. Voir par exemple P. Suppes, «A Comparison of the Meaning and Use of Models in Mathematics and the Empirical Sciences », in H. Freudenthal (éd.), The Concept and the Role of the Model in Mathematics and Natural and Social Sciences, Reidel, Dordrecht, Neth., 1961 ; P. Suppes, «What is a Scientific Theory? », in S. Morgenbesser, Philosophy of Science Today, Basic Books, New York, 1967 ; F. Suppe, The Semantic Conception of Theories and Scientific Realism, University of Illinois Press, Urbana et Chicago, 1989 ; B.V. Fraassen, The Scientific Image, Clarendon Press, Oxford, 1980 ; R. Giere, Science Without Laws, University of Chicago Press, 1999.

5. La notion de modèle est ici à comprendre dans le sens qu'elle a dans le cadre de la théorie des modèles issue des travaux de Tarski : un modèle est une interprétation qui satisfait un certain ensemble d'axiomes, c'est-à-dire un ensemble d'objets dont les relations sont décrites par ces axiomes.

6. Ronald Giere parle plus volontiers de « ressemblance» (cf. R. Giere, Explaining Science. A Cognitive Approach, The University of Chicago Press, 1988) ; certains défenseurs récents de cette conception ont proposé des variantes de la notion d'isomorphisme, en parlant d' «homomorphisme » ou d' «isomorphisme partiel» (voir par exemple N. Da Costa et S. French, «The Model-Theoretic Approach in the Philosophy of Science, Philosophy of Science, vol. 57 , n², pp. 248-265, 1990). Cela n'a pas d'importance pour le présent propos.

7. Plus précisément, les modèles théoriques sont isomorphes aux «modèles de données », c'est-à-dire aux données une fois décrites et mises en forme de manière à ce que les modèles mathématiques de la théorie puissent s'y appliquer (cf. P. Suppes, «Models of data », in E. Nagel, P. Suppes et A. Tarski (éd.), Logic, Methodology and Philosophy of Science. Proceedings of the 1960 International Congress, Stanford University Press, p. 252-261, 1962).

8. Sur ce point, P. Suppes, 1968, «The Desirability of Formalization in Science », The Journal of Philosophy, vol. 65, n²0, p. 651-664, est particulièrement explicite. 
ne chercherai pas à faire ici - ne permet pas d'expliquer la manière dont les modèles, qu'ils incarnent des structures mathématiques ou non, sont utilisés, en pratique, dans les raisonnements des scientifiques. Comme l'ont montré plusieurs critiques de la conception sémantique ${ }^{9}$, l'isomorphisme, la ressemblance et autres relations symétriques de ce type ne permettent pas d'expliquer l'aspect intentionnel de la représentation et la manière dont une représentation permet à un sujet cognitif de tirer des inférences à propos de l'objet représenté.

Dans cette perspective, je propose d'étudier l'activité théorique et plus précisément, ici, le problème de la représentation scientifique en prêtant attention au type d'opérations cognitives et parfois de manipulations concrètes effectuées par les scientifiques lors de l'apprentissage, du développement et de l'application des théories. Cela implique de s'intéresser non pas aux théories prises comme des touts, mais aux instruments représentatifs de diverses sortes que les scientifiques utilisent effectivement pour prédire et expliquer les phénomènes; ces instruments représentatifs sont généralement appelés «modèles» et sont de nature très variée : diagrammes, graphes, maquettes en trois dimensions, modèles imaginaires comme le pendule simple en mécanique classique, etc.

Dans cet article, je chercherai donc à répondre à la question suivante : comment les scientifiques utilisent-ils les modèles pour représenter les phénomènes ? Cela implique de se demander en vertu de quelles propriétés un modèle permet à un agent cognitif de tirer des inférences à partir de lui pour obtenir des informations concernant le système représenté.

Je concentrerai mon attention sur un type particulier d'objets que l'on appelle couramment «modèles », à savoir les modèles imaginaires - que j'appellerai «modèles-I »- comme le pendule simple. L'importance de cette sorte de modèles a souvent été soulignée ${ }^{10}$; on a en effet très couramment affaire à eux dans l'enseignement, la théorie et la pratique scientifiques. Ces modèles posent un problème tout particulier dans la mesure où il ne s'agit pas d'outils de représentation concrets comme des équations ${ }^{11}$ ou des diagrammes, sans qu'ils soient pour autant réductibles aux structures

9. Roman Frigg, 2002, «Models and representation: why structures are not enough », in Measurement in Physics and Economics Project Discussion Paper Series, DP MEAS 25/02, London School of Economics ; Roman Frigg, 2008, «Models and Fictions », in Synthese; Mauricio Suárez, 2003, «Scientific representation : against similarity and isomorphism », in International Studies in the Philosophy of Science, vol. 17, ${ }^{\circ} 3$, p. 225-244.

10. Voir R. Frigg (2002 et 2008), P. Godfrey-Smith, «The strategy of model-based science », Biology and Philosophy, p. 725-740, 2006 ; R. Giere, 1988, R. Giere, Scientific Perspectivism, The University of Chicago Press, 2006.

11. Une équation n'est certes pas un objet concret au sens où des marques sur un papier le sont : c'est plutôt une classe ou un type de marques (token). Il en va de même pour un diagramme, d'ailleurs. Cependant, comme il apparaîtra plus clairement avec la définition que je propose de la notion de format, équations et diagrammes sont des représentations formatées et chaque équation ou diagramme correspond à ce que j'appelle plus bas des «types de représentation». En cela, ils se distinguent nettement d'un objet imaginaire comme le pendule. 
mathématiques dont ils sont parfois des instances. Autrement dit, ils ne sont ni des objets mathématiques abstraits ni des marques concrètes tenant lieu d'autre chose. Le pendule simple n'existe pas, mais s'il existait, il aurait des propriétés qui ne sont pas simplement celle de la structure mathématique qu'il incarne : il aurait un balancier, un fil, une composition moléculaire, etc. Roman Frigg ${ }^{12}$ distingue deux ensembles de questions concernant les modèles-I (qu'il appelle «systèmes modèles ») : les questions ontologiques qui consistent à se demander à quel type d'entités ces modèles appartiennent, et les questions sémantiques concernant le type de relations que les modèlesI entretiennent avec les phénomènes qu'ils représentent. Frigg s'attaque au premier problème comme un préalable nécessaire pour pouvoir résoudre le second. J'emprunte une route différente, et j'affronte ici le problème sémantique, en le posant de la façon suivante : comment les scientifiques utilisent-ils les modèles-I pour tirer des inférences et acquérir des connaissances sur les systèmes qu'ils représentent?

Premièrement, je propose une clarification de la notion même de représentation (2.1) en insistant sur l'importance de ce que j'appelle le « format» d'une représentation (2.2) pour les inférences que les agents cognitifs peuvent en tirer. Ensuite, je propose une réponse à la question posée ci-dessus en analysant les différentes relations de représentations qui sont en jeu dans l'usage des modèles-I à l'aide du nouvel outil d'analyse qu'est la notion de format (3.1 et 3.2). Cette mise en œuvre de la notion de format pour traiter un problème actuellement débattu me permet finalement de suggérer que l'étude de l'activité théorique et de l'utilisation des représentations scientifiques en général gagneraient à prêter attention à la diversité des formats qui sont utilisés dans la pratique scientifique, en parallèle d'une approche logique et abstraite de la structure des théories.

\section{Formats de représentation}

Pour commencer, je souhaiterais présenter ici la conception de la représentation qui sous-tendra mon analyse dans la section 3. J'introduirai dans cette section une nouvelle notion, celle de «format », qui est destinée à nous aider à comprendre certains des phénomènes importants en jeu dans l'utilisation des représentations par des agents cognitifs qui cherchent à en tirer des informations sur les choses représentées. C'est un préliminaire nécessaire à l'étude de l'utilisation des modèles-I dans la représentation de systèmes physiques, que j'entreprendrai dans la section 3 .

12. R. Frigg, 2008. 


\subsection{Représentation}

Je propose de définir la représentation comme une activité cognitive $\mathrm{e}^{13}$ consistant à utiliser un instrument (l'objet représentant, ou representans) dans le but d'acquérir des connaissances à propos de quelque chose dont il tient lieu (le representatum). Dans les cas les plus simples et les moins problématiques, le representans est un objet concret particulier, comme des marques sur un papier (une figure ou des signes linguistiques), des vibrations de l'air, ou tout autre chose à laquelle il est possible d'avoir un accès perceptuel direct ${ }^{14}$. Le representatum peut être un objet physique, certaines propriétés d'un objet, l'évolution temporelle d'une valeur, un processus causal, etc. Je parlerai de manière neutre de la «scène » représentée.

Les propriétés perceptuelles du representans sont des signaux portant des informations concernant le representatum. Le terme «information », ici, doit être compris comme désignant tout contenu propositionnel pouvant être objet de croyance. Ainsi, «contenu informationnel» est synonyme de «contenu propositionnel». La conception de la représentation que je propose ici ne se restreint pas aux représentations scientifiques en tant que telles ; cependant, je m'intéresse exclusivement à la représentation en tant que moyen d'acquérir des connaissances - scientifiques ou non - et par conséquent je restreins mon analyse au contenu propositionnel informationnel - des représentations ${ }^{15}$.

Afin d'acquérir des connaissances sur le representatum à l'aide du representans, un agent cognitif doit maîtriser les règles d'interprétation de ces signaux. En effet, un même ensemble de marques peut être utilisé pour représenter une scène en vertu de plusieurs - en fait, d'une infinité de - types de relations entre ses propriétés perceptuelles et les propriétés de la scène. Tout dispositif peut être déclaré représenter n'importe quelle scène par fiat. Les relations de représentation peuvent aussi être fondées sur des relations non conventionnelles entre le representatum et le representans : par exemple, la photographie d'un chien représente ce chien - sous certaines

13. Le terme «représentation» souffre d'une ambiguïté difficile à éliminer par des conventions linguistiques : il peut en effet désigner la pratique cognitive qui consiste à utiliser un objet pour en représenter un autre ; il peut également désigner l'objet concret tenant lieu de la chose représentée. Comme il apparaîtra dans ce qui suit, je désignerai cette dernière par le terme de «representans » (on pourrait, par souci de légèreté, choisir le terme assez neutre de «figure », mais il n'est pas suffisamment englobant, car il n'inclut pas les signes linguistiques) et je réserverai le terme de «représentation » à la pratique cognitive, sauf dans les cas où le contexte suffira à dissiper toute ambiguïté.

14. Parler d' «accès perceptuel direct» n'implique pas un engagement pour une théorie déterminée de la perception ; je pense qu'il y a un sens dans lequel on peut dire, sans que cela soit sujet à controverse, que j'ai un accès perceptuel direct aux objets physiques concrets qui m'entourent - une connaissance par «accointance »- , différent de la connaissance que j'ai d'objets imaginaires ou abstraits comme les structures mathématiques.

15. Par exemple, je ne m'intéresserai pas ici au contenu expressif des représentations, dans le sens goodmanien d' «expression » (Nelson Goodman, 1968/1976, Langages de l'art, trad. J. Morizot, éd. Jacqueline Chambon, 1990, chapitre 2 ; à partir de maintenant, les numéros de pages correspondent à celles de la traduction française). 
conditions de luminosité et de cadrage, et pourvu qu'elle soit prise depuis une distance adéquate et avec une mise au point correcte - en vertu de la relation causale entre la lumière reflétée par le chien et l'exposition du film. Ce type de relation de représentation dépend certes aussi de plusieurs paramètres conventionnels, mais la relation causale joue un rôle fondamental dans l'établissement de la relation de représentation entre la photographie et le chien.

Nelson Goodman ${ }^{16}$ conçoit les relations entre n'importe quel representans et son representatum comme des relations de dénotation, gouvernées par un système symbolique spécifique. La dénotation est une relation entre un symbole et un objet du monde. Une image représentant un chien entretient le même type de relation avec les chiens réels qu'une occurrence du mot «chien ». Un système symbolique consiste en une syntaxe et une sémantique: la syntaxe est l'ensemble des propriétés perceptuelles (les «marques ») qui comptent comme des signaux portant des informations, jointes à leurs règles de combinaison, et la sémantique établit la relation entre ces propriétés et ce qu'elles dénotent. Selon le système symbolique qu'on utilise pour l'interpréter, le même ensemble de marques peut dénoter des scènes très différentes. Par exemple, « des lignes noires en zigzag sur fond blanc » peuvent être un «fragment d'électrocardiogramme » représentant des battements de cœur dans un système diagrammatique, aussi bien qu'un «dessin du mont Fujiyama ». Les propriétés perceptuelles pertinentes des deux figures sont différentes : «Les seuls traits pertinents du diagramme sont l'ordonnée et l'abscisse de chacun des points que traverse le centre de la ligne. L'épaisseur de la ligne, sa couleur et son intensité, la grandeur absolue du diagramme, etc., n'importent pas », alors que tout changement dans ces propriétés est significatif dans le cas de l'esquisse ${ }^{17}$.

J'adopte globalement cette perspective goodmanienne sur la représentation; cependant, je vais proposer une nouvelle notion, celle de «format», dans le but de saisir certains aspects cognitifs et pragmatiques de l'utilisation des représentations par les humains, dont la notion de système symbolique ne rend pas compte.

\subsection{Formats}

S'il maîtrise le système symbolique adéquat à l'interprétation d'un certain representans, un sujet peut extraire des informations concernant le representatum. Cependant, deux representans ${ }^{18}$ peuvent contenir exactement la même information (à propos du même objet) tout en nous la transmettant de manière différente. Prenons par exemple une photographie numérique et le code numérique pour chacun de ses pixels: ils sont strictement équivalents du point de vue de l'information contenue. Cependant, seule une représentation ordonnée et différenciée des pixels dans une image à deux

16. N. Goodman, 1968/1976.

17. N. Goodman 1968/1976, p. 273.

18. Dans un souci de clarté, je renonce ici au devoir d'accorder en nombre les termes latins. 
dimensions permet à un sujet humain sans aide extérieure d'extraire des informations concernant la scène photographiée. Le contenu informationnel du code numérique, sans outil informatique externe le transformant en une image bi-dimensionnelle, nous est strictement inaccessible.

Il existe des cas moins extrêmes de représentations équivalentes du point de vue de l'information mais différentes du point de vue de leur contenu cognitivement accessible. Par exemple, les résultats d'un relevé de températures en différents endroits de la planète peuvent être présentés sous la forme d'une liste de triplets de chiffres, les deux premiers indiquant les coordonnées des différents points auxquels les relevés ont été effectués et le troisième la valeur correspondante de la température; les mêmes données peuvent être présentées sous la forme d'une carte en deux dimensions, sur laquelle les emplacements des triplets de chiffres respectent les distances relatives entre les points de la planète où les relevés ont été effectués. On peut éventuellement colorier les différentes zones par des teintes correspondant aux fourchettes de températures. L'information contenue dans les deux représentations est exactement la même, et les agents humains contrairement à ce qui se passe dans le cas de l'image digitale - sont capables de l'extraire de n'importe laquelle des deux. Mais la carte rend certaines informations plus aisément disponibles: par exemple, si les couleurs chaudes correspondent aux températures élevées et les couleurs froides aux basses températures, on peut rapidement conclure que la partie méridionale de la zone représentée est plus chaude que sa partie septentrionale. Extraire une telle information de la seule liste de chiffres requiert plusieurs étapes inférentielles. En outre, les opérations inférentielles conduisant à la même conclusion à partir de la considération des deux représentations n'est pas du même type : lire une liste de valeurs numériques et les comparer en prenant en compte les coordonnées correspondantes ne consiste pas en la même opération cognitive que de comparer les emplacements spatiaux de différentes couleurs sur une carte.

De manière plus générale, on peut affirmer que dans toute représentation, certaines informations sont immédiatement disponibles ${ }^{19}$ tandis que d'autres ne peuvent être extraites que par le biais de processus inférentiels plus ou moins longs et de types variés ${ }^{20}$.

19. Il ne s'agit pas ici de la question de savoir s'il existe un type de connaissance perceptuelle qui ne serait le fruit d'aucun processus inférentiel - qui serait authentiquement immédiat. En parlant d' « information immédiatement accessible », par opposition à l'information obtenue par le biais d'inférences, je n'affirme pas qu'il y ait une différence de nature plutôt que de degrés entre les deux. Imaginons qu'il y ait de l'eau sur le pavé tandis que je marche dans la rue ; je dirais que la proposition «il y a de l'eau» est une information immédiatement accessible, tandis que «il a plu» est obtenu par un processus inférentiel. Je n'entrerai pas dans plus de détails ici, mais la notion intuitive d'immédiateté est suffisante. John Kulvicki, 2008, «Knowing with images » (manuscrit non publié) propose une élaboration de cette notion ; c'est également de ce manuscrit qu'est inspiré l'exemple des relevés de température.

20. Des études en intelligence artificielle sur les capacités de résolution de problèmes par des agents humains (voir H. Simon et J. Larkin, "Why a diagram is (sometimes) worth ten thousand words », Cognitive Science, vol.11, p.65-99, 1987, pour un traitement 
La notion de format est destinée à saisir ces différences cognitives ou inférentielles ${ }^{21}$. On peut certes, dans des termes goodmaniens, affirmer que ces différentes représentations doivent être interprétées dans différents systèmes symboliques: leurs règles syntaxiques et sémantiques sont très différentes. Mais mon but est de caractériser et de mesurer les différences entre ces représentations dans la perspective pragmatique et cognitive de l'agent qui est censé tirer des inférences à partir du representans pour acquérir des connaissances sur le representatum. En conséquence, la notion de format doit être relative au contexte et à l'agent, tandis que la notion de système symbolique peut être entièrement définie en termes objectifs de syntaxe et de sémantique.

Une première définition, informelle, de la notion de format, serait la suivante: le format d'une représentation est la manière dont cette représentation transmet l'information qu'elle contient à l'usager. L'image digitale et ses lignes de code sont présentées dans des formats différents; il en va de même pour la liste de chiffres représentant différentes valeurs de température et la carte correspondante. Plus précisément, le format $F_{R S}$ d'un ensemble particulier de marques $R$ représentant une scène $S$ peut être isolé en fonction du rôle inférentiel ${ }^{22}$ de la représentation, qui consiste en :

- $\quad I$ : le type et la quantité d'information sur $S$ qu'un agent particulier $A$ dans un contexte donné $C$ peut tirer de $R$;

- $\quad C C$ : la longueur relative du processus inférentiel $P$ - ou du nombre d'étapes inférentielles, si elles peuvent être comptées - nécessaires à $A$ dans $C$ pour tirer $I$ de $R$ (le coût cognitif) ;

- $C K$ : le type d'opérations cognitives incluses dans $P$.

philosophique de la question) ont montré comment la présentation de données dans une image en deux dimensions peut augmenter considérablement les capacités inférentielles des agents. Parfois, de telles considérations sont accompagnées d'hypothèses cognitives concernant le format des représentations mentales et des raisonnements (voir Johson-Laird, «Mental models. Towards a cognitive science of language, inference and consciousness », Cognitive Science Series, Harvard University Press, 1983). Cependant, de telles hypothèses ne sont pas nécessaires pour affirmer que le mode de présentation des données a une influence sur les capacités humaines de raisonnement.

21. Je parle indifféremment d'équivalence et de différence cognitives ou inférentielles. Bien qu'il existe des processus cognitifs qu'on ne caractérise pas d'inférentiels, tous les processus cognitifs qui m'intéressent ici sont de type inférentiel, dans la mesure où je me concentre exclusivement sur le contenu propositionnel des représentations.

22. Une telle conception de la représentation trouve un fondement théorique dans un ensemble de thèses en philosophie du langage et de l'esprit, regroupées sous le nom de «sémantique des rôles conceptuels ». Cette perspective est défendue entre autres par Gilbert Harman, Ned Block, Hartry Field et Paul Horwich (voir notamment M. Greenberg et G. Harman, «Conceptual Role Semantics », UCLA School of Law : UCLA Public Law Series, pp. 5-16, 2005). La sémantique des rôles conceptuels identifie le contenu d'une représentation (la signification d'un terme ou le contenu d'un concept, selon qu'on l'utilise comme une théorie du langage ou de l'esprit) à son rôle inférentiel dans la vie cognitive des individus. 
Tout cela dépend certes de la syntaxe et de la sémantique du système sous lequel $R$ doit être interprétée, mais cela ne suffit pas. $F_{R S}$ dépend aussi de $A$ et de $C$ : des capacités cognitives, des talents et de la connaissance d'arrière-plan de l'agent, de ses buts cognitifs (l'information recherchée), de l'information disponible au préalable, etc. En effet, selon les capacités cognitives et les buts de l'agent, un changement dans les propriétés perceptuelles ne compte pas nécessairement comme un changement de format, dans la mesure où il ne modifie pas nécessairement le rôle inférentiel de la représentation : par exemple, dans un graphe représentant l'évolution temporelle d'un pendule, les propriétés pertinentes sont les coordonnées des points représentant sa position. La couleur de la ligne n'importe pas : que la ligne soit noire ou bleue, $I, C C$ et $C K$ sont inchangés ; par conséquent le format est le même. En revanche, si l'on cherche à représenter l'évolution temporelle de deux pendules ayant des fréquences d'oscillation différentes, il peut s'avérer utile de tracer les deux lignes dans des couleurs différentes. Le graphe bicolore facilite certaines inférences et n'est par conséquent pas dans le même format que le graphe unicolore - tout en étant dans un format très proche. En revanche, si l'on dessine un graphe représentant l'évolution temporelle de la position d'un pendule en utilisant plusieurs couleurs, de manière arbitraire, ou correspondant à des différences non pertinentes du point de vue de l'information recherchée (par exemple aux variations de température dans la pièce), le format change de nouveau, car la couleur crée une confusion inutile qui peut rendre les inférences concernant le mouvement du pendule moins aisées que dans le cas du graphe unicolore.

Insistons sur un point : le format étant relatif à l'agent, la différence entre un graphe bicolore et un graphe unicolore ne vaudrait pas nécessairement, par exemple, pour un sujet daltonien. De même, une équation différentielle n'est pas dans le même format pour un mathématicien entraîné et un débutant. Pourquoi insister ainsi sur la relativité de la notion de format au sujet ? Dans l'analyse qui suit de l'utilisation des modèles-I, je ne ferai pas mention des différences entre agents individuels, supposant implicitement un scientifique moyen avec des capacités cognitives normales. Cependant, ces précisions sont importantes, au moins pour deux raisons différentes. La première est que la notion de format est destinée à rendre compte des aspects cognitifs de l'interprétation des représentations et est donc nécessairement relative au sujet, contrairement à la notion de système symbolique. Il est difficile, une fois que l'on fait intervenir les capacités cognitives humaines, de tracer une distinction nette entre des différences épistémiques qui vaudraient pour tous et seraient par conséquent « objectives » et des différences psychologiques dépendantes de chacun. La deuxième raison est que cela permet d'appliquer la notion de format à des cas différents de celui que je traite dans ce qui suit, pour lesquels les différences individuelles comptent. Par exemple, dans une étude de la vulgarisation ou de l'enseignement scientifiques, il peut être utile, dans une perspective normative, de caractériser quel serait le bon format - celui qui minimiserait le coût cognitif en fonction de l'information que l'on cherche à 
transmettre et des capacités cognitives du public. Un autre cas où la notion de format et sa relativité au sujet permettent de saisir des phénomènes importants de l'activité théorique auxquels les analyses philosophiques classiques ne rendent pas vraiment justice est celui de l'utilisation d'un même type de représentations par différents scientifiques qui ne partagent pas les mêmes engagements théoriques et qui les interprètent par conséquent différemment ${ }^{23}$.

Certes, $I, C C$ et $C K$ sont impossibles, en pratique, à mesurer d'une manière précise. Cependant, on peut comparer deux représentations en affirmant que, par exemple, extraire telle information de l'une exige un coût cognitif plus grand que de l'autre, ou encore que telle information qui est accessible dans l'une est pratiquement inaccessible dans l'autre. De même, malgré le fait que l'on n'ait encore aucune description précise qui fasse consensus du format des représentations et des processus mentaux, on peut intuitivement reconnaître qu'extraire des informations d'un graphe d'une part et d'une équation d'autre part ne consiste pas dans le même type de processus cognitif. La notion de format est principalement destinée à être un outil de comparaison entre différentes représentations de la même scène, qui sont au moins partiellement équivalentes informationnellement, ou entre différents usages d'un même ensemble de marques par différents agents.

Pour finir, je définis un type de représentation comme la classe de tous les dispositifs représentationnels actuels et possibles de la même scène dans le même format. En d'autres termes, un type de représentation est identifié sur la base de son rôle inférentiel : c'est la classe de tous les representans qui permettent à des agents à capacités cognitives égales dans des contextes équivalents de tirer exactement les mêmes informations par des processus inférentiels semblables.

\section{Comment utilise-t-on les modèles-I pour représenter des systèmes physiques ?}

Venons-en à présent à la question principale que j'ai posée en introduction: comment utilise-t-on des modèles-I pour représenter des systèmes physiques? Les modèles-I sont très utilisés par les scientifiques dans le développement de leurs hypothèses ainsi que dans la prédiction et l'explication des phénomènes; ils jouent aussi un rôle central dans l'enseignement scientifique. Dans les manuels, les lois scientifiques sont la plupart du temps introduites par la référence à un modèle-I qui permet aux étudiants de comprendre ce que la loi signifie. Quand il s'agit de prédire et

23. Je ne peux pas développer d'exemple particulier ici ; cependant, c'est un phénomène fréquent dans l'histoire des sciences. Un exemple célèbre en est l'interprétation et l'utilisation différentes des diagrammes de Feynman par Richard Feynman d'une part et Freeman Dyson de l'autre (voir D. Kaiser, Drawing Theories Apart. The Dispersion of Feynman Diagrams in Postwar Physics, University of Chicago Press, 2005). 
d'expliquer le comportement d'un système physique, on en appelle souvent à un modèle-I, affirmant qu'il s'applique, moyennant plusieurs approximations et idéalisations, au système physique: par exemple, le mouvement du balancier d'une horloge normande peut être représenté à l'aide du modèle du pendule simple. Il existe beaucoup d'autres exemples de modèles-I dans plusieurs domaines scientifiques ; on peut citer, par exemple, le modèle des boules de billard pour l'étude du mouvement moléculaire dans le cadre de la théorie cinétique des gaz, les populations isolées en génétique des populations, et les agents parfaitement rationnels en économie.

Je propose de caractériser les modèles-I comme les référents imaginaires de lois scientifiques : ils n'existent pas dans le monde extérieur, mais, s'ils existaient, leur comportement serait une instance parfaite de ces lois. Certains philosophes des sciences, comme Roman Frigg ${ }^{24}$, affirment que les modèles-I (les «systèmes modèles ») sont des fictions ${ }^{25}$. Bien que je sois globalement en accord avec cette idée, mon objet central est de comprendre comment nous utilisons ces modèles pour représenter. Or, comme il apparaîtra dans ce qui suit, une telle question peut être abordée sans qu'il soit nécessaire d'adopter une théorie particulière des fictions. Je resterai par conséquent agnostique sur ce point.

Gardant présent à l'esprit que le rôle principal d'un modèle est de nous permettre de tirer des inférences concernant le système qu'il représente, on peut se demander en vertu de quelles caractéristiques les modèles-I sont utilisés comme des représentations. Doit-on leur appliquer une sémantique spéciale ? Autrement dit, les relations entre les propriétés du modèle et celles du système, en vertu desquelles le premier est une représentation du second, sont-elles d'un type spécial ? Les propriétés mêmes du modèle-I qui en font une représentation sont-elles propres à ce type d'entités ?

Ma stratégie dans ce qui suit va consister à analyser et clarifier les différentes relations de représentation qui sont en jeu dans l'utilisation d'un modèle-I - celui du pendule simple - en m'appuyant sur la conception de la représentation que j'ai proposée en section 2. En effet, il existe au moins deux relations de représentations dans ce que j'ai exposé précédemment. Premièrement (3.1), dans la mesure où le pendule simple n'existe pas dans le monde physique extérieur (il n'existe pas de fil sans masse ni de masse sans volume), on ne peut en avoir une connaissance directe, par « accointance »; le pendule simple doit donc être donné par le biais d'une représentation d'une certaine sorte. Dans ce cas, je parlerai de « caractérisation » plutôt que de «représentation», pour des raisons que je préciserai dans la prochaine section. Deuxièmement (3.2), le système physique étudié est représenté à l'aide du pendule simple. Dans le premier cas, le modèle-I est l'objet de la représentation - ou plus précisément de la caractérisation - et dans le second cas il est l'instrument de représentation (le representans). Je vais montrer

\section{R. Frigg, 2008.}

25. R. Giere (2006), qui s'intéresse aussi à cette sorte de modèles, refuse de parler de fictions, et préfère caractériser ces modèles comme des « objets abstraits ». 
que la clef du pouvoir représentationnel des modèles-I réside dans la relation entre ces deux processus de représentation, en mettant en œuvre le nouvel outil d'analyse proposé dans la section précédente, la notion de format.

\subsection{Caractérisations du modèle-I}

Dans ce paragraphe, mon objet est de mettre en lumière le fait qu'on ne peut pas accéder à un modèle-I - par exemple, le pendule simple - sans qu'il soit donné dans un format particulier, et que le même modèle-I peut être caractérisé dans différents formats, plus ou moins appropriés à l'information que l'on cherche à obtenir. Dans la mesure où le pendule simple n'existe pas dans le monde spatio-temporel, on y accède par le biais de nos capacités de représentations comme, par exemple, le langage ${ }^{26}$. Suivant Nelson Goodman, et pour plus de clarté, je ne parlerai pas de «représentation »du pendule simple, réservant « représentation » pour les cas où le representatum est un objet existant et non fictif ou imaginaire. Je parlerai plutôt de « caractérisation ${ }^{27}$ du pendule simple.

Le pendule simple est introduit dans la plupart des manuels de mécanique comme le référent de la loi du mouvement oscillatoire (oscillateur harmonique). Il est défini comme une masse ponctuelle $m$ fixée à l'extrémité inférieure d'un fil inextensible sans masse et de longueur $l$, qui peut bouger librement autour de son extrémité supérieure fixe.

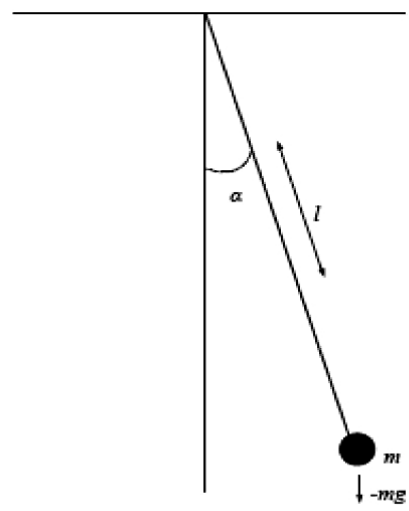

Figure $1:$ dessin schématique du pendule simple

26. Dans la mesure où je ne prends ici parti pour aucune théorie particulière de la fiction, je ne prétends pas que le pendule soit construit par nos capacités représentationnelles ; cependant, quel que soit son statut ontologique, on ne peut y accéder que par le biais de caractérisations concrètes.

27. En termes goodmaniens, les caractérisations de pendule sont des «représentations-dependules »[pendulum-representations]. 
Dans le cadre théorique de la mécanique classique, on peut décrire le mouvement du balancier du pendule par l'équation suivante (1), avec $x$ représentant la position, a l'accélération ${ }^{28}$ et $g$ la force gravitationnelle.

$$
m \mathbf{a}=-\left(\frac{m g}{l}\right) x \cos (\alpha)
$$

Pour des oscillations de petite amplitude, l'angle d'oscillation $\alpha$ est assez petit pour que l'on puisse considérer $\cos (\alpha) \operatorname{comme}$ égal à un. On obtient alors l'équation suivante (2) :

$$
m \mathbf{a}=-\left(\frac{m g}{l}\right) x
$$

Il s'agit d'une équation différentielle décrivant un mouvement oscillatoire linéaire. Si l'on n'effectuait pas l'approximation mentionnée, le mouvement décrit ne serait pas linéaire.

Les solutions de cette équation pour la position $x(t)$ et pour la vitesse $v(t)$ en fonction du temps ont une forme harmonique représentée par la fonction :

$$
f(t)=A \cos \left[\left(\sqrt{\frac{g}{l}}\right) t\right]+B \sin \left[\left(\sqrt{\frac{g}{l}}\right) t\right]
$$

où $A$ et $B$ sont des constantes à déterminer en fonction des conditions initiales du système représenté.

En introduisant les conditions initiales dans la fonction ci-dessus, on peut dessiner un graphe pour la position et pour la vitesse de $m$ en fonction du temps. Les deux graphes ont une forme sinusoïdale. Pour les conditions initiales $x_{(t=0)}=A$ et $v_{(t=0)}=0$, on obtient les graphes de la figure $2^{29}$.

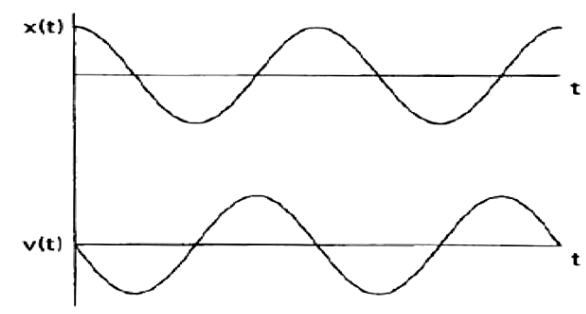

Figure 2 : position $(x)$ et vitesse $(v)$

en fonction du temps $t$ de la masse $m$ du pendule simple (tiré de R. Giere, 1988, p. 69)

28. Suivant un usage répandu, j'utilise des caractères gras pour représenter des quantités vectorielles.

29. Ces figures sont empruntées à R. Giere, 1988. Dans ce chapitre, ces graphes représentent la position et la vitesse d'un système masse-ressort. Mais, une fois l'approximation de l'angle d'oscillation du pendule à zéro effectuée, l'équation pour les deux systèmes est la même. 
Dans certains cas, il est avantageux de décrire le mouvement du pendule en termes d'énergie, plutôt que de force. Les équations du mouvement pour le système peuvent être écrites à l'aide de l'hamiltonien $H$ qui est la somme des énergies potentielle et cinétique du système :

$$
\frac{d x}{d t}=\frac{D H}{D p}
$$

et

$$
\frac{d p}{d t}=-\frac{D H}{D x}
$$

Les solutions de ces équations sont exprimées en termes de position $x(t)$ et de quantité de mouvement $p(t)^{30}$. En prenant $x$ et $p$ comme les axes d'un espace d'états euclidien en deux dimensions, l'état du système à chaque instant est représenté par un point unique, et son évolution est représentée par une ellipse, comme dans la figure ci-dessous.

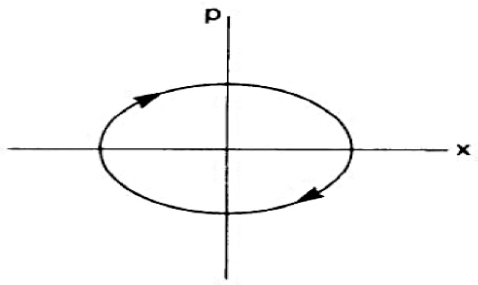

Figure 3 : état d'un pendule simple dans un espace position-quantité de mouvement (tiré R. de Giere, 1988, p. 72)

Les équations et les graphes que je viens de présenter sont des caractérisations mécaniques de l'évolution temporelle du pendule simple dans plusieurs formats. En effet, la notion de format nous permet de distinguer aussi bien entre une représentation linguistique (équation) et une représentation spatiale (graphe) qu'entre deux représentations linguistiques (deux équations dans des langages mathématiques différents) et entre deux représentations spatiales (dans des systèmes de coordonnées différents). Les différences entre formats sont de degrés divers et se produisent selon différentes dimensions. La distance entre formats pourrait être conçue comme une fonction du coût cognitif nécessaire à tirer une représentation dans un format à partir d'une représentation en un autre format; la dimension selon laquelle un changement de format peut se décrire peut être conçue comme dépendant du type d'opération cognitive qui est nécessaire : par exemple, tracer un graphe à partir d'une équation n'est pas le même type d'opération cognitive que de projeter un graphe dans un système de

30. La quantité de mouvement $p$ d'un système physique est définie comme le produit de sa masse et de sa vitesse $(m v)$. 
coordonnées sur un autre système de coordonnées. Les deux sont des changements de format, selon différentes dimensions.

Le graphe de la figure 2 est tiré de la fonction (3): il représente les valeurs qu'elle prend dans certaines conditions ; présenter ces solutions dans un format de graphe, bien que n'ajoutant rien à leur contenu informationnel, rend cependant certaines informations immédiatement accessibles à l'agent, qui devrait faire de nombreuses inférences pour les tirer de la considération des solutions présentées dans un format linguistique.

La différence de format entre les équations newtonienne et hamiltonienne n'est pas du même type: les deux sont des formats linguistiques, et elles sont mathématiquement équivalentes, dans la mesure où on peut montrer qu'elles sont réciproquement déductibles. Cependant, elles sont différentes d'un point de vue inférentiel. Elles ne sont pas utilisées dans les mêmes cas et elles ne facilitent pas les mêmes inférences. Utiliser un format ou l'autre ne conduit pas à procéder aux mêmes opérations cognitives: résoudre un problème dans le cadre newtonien implique d'utiliser des équations différentielles du second ordre dont les solutions sont exprimées en termes de position et de vitesse - et qui n'ont dans certains cas pas de solution analytique -, tandis que les équations hamiltoniennes sont des équations du premier ordre et leurs solutions sont exprimées en termes de position et de quantité de mouvement. En outre, les équations hamiltoniennes révèlent les relations profondes entre la mécanique classique et d'autres champs de la physique - comme la mécanique statistique, la mécanique quantique et la mécanique quantique relativiste - dont les équations centrales sont dans des formats proches de celui des équations hamiltoniennes en mécanique classique.

La considération des graphes respectivement tirés des formats linguistiques newtonien et hamiltonien révèle clairement les différences entre eux. Elle fait également apparaître leur équivalence : on peut en effet comprendre le lien entre l'espace d'état de la figure 3 et les solutions présentées dans la figure 2 en imaginant un troisième axe, représentant le temps, perpendiculaire au plan $x-p$. L'état du système en fonction du temps est représenté par une spirale de forme elliptique tournant autour de l'axe $t$, comme dans la figure ci-dessous.

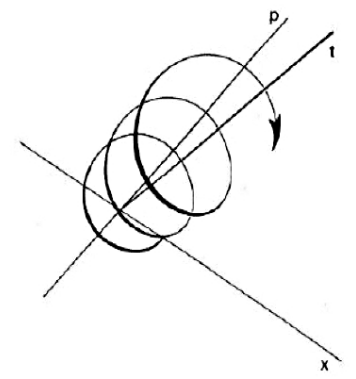

Figure 4 : état d'un pendule simple dans un espace position-quantité de mouvement-temps (tiré de R. Giere, 1988, p. 73) 
La projection de cette trajectoire sur les plans $x$ - $t$ et $p$ - $t$ donne les fonctions sinusoïdales dont les graphes étaient présentés dans la figure 2. De même, la projection de l'état de l'espace $x$ - $t-p$ sur le plan $x$ - $p$ donne l'ellipse de la figure $3^{31}$. Cela met en lumière les différentes dimensions selon lesquelles un changement de format peut se produire et les différentes inférences que de tels changements facilitent.

Notons ici la forte analogie entre cette notion de format et la notion de "gabarit ${ }^{32}$, telle que Paul Humphreys ${ }^{33}$ la propose. Un gabarit est un schéma syntaxique de calcul - le plus souvent, une équation - qui est indépendant des théories, au sens où l'on peut trouver différents gabarits au sein d'un même cadre théorique, et où le même gabarit peut se retrouver dans différents domaines. Les équations newtonienne et hamiltonienne sont typiquement des gabarits différents au sein du champ de la mécanique; parfois, un changement de gabarit - comme un changement de coordonnées - conduit à un changement dans la calculabilité de l'équation; en outre, le gabarit hamiltonien est aussi utilisé dans d'autres domaines. Ainsi, les gabarits ne sont pas attachés à une théorie déterminée, en tant qu'elle serait définie par le domaine des phénomènes qu'elle explique, mais ils incarnent bien plutôt des formes de computation. Le but de Humphreys est d'attirer l'attention des philosophes des sciences sur la pratique et la calculabilité, en contraste avec des approches purement logiques des théories et des modèles, qui considèrent comme équivalentes des formulations qui en pratique ne conduisent pas au même type de calculs et ne sont pas également utilisables. Cette perspective a contribué à motiver mon élaboration de la notion de format. Il y a cependant des différences importantes entre gabarits et formats, et entre la perspective de Paul Humphreys et la mienne : la notion de gabarit est destinée à rendre justice, dans une science où les ordinateurs et les simulations numériques prennent une place de plus en plus centrale, à l'importance de ce qui est, en pratique, faisable, calculable, par opposition à une épistémologie fondée sur les catégories classiques de théories et d'expérience. Un changement de gabarit est un changement dans la difficulté d'un problème et dans la possibilité même pour un ordinateur d'y apporter des solutions. Cela ne concerne pas les agents cognitifs. La notion de format, elle, est destinée à rendre compte des différences cognitives dans l'utilisation des représentations par des agents humains.

J'ai montré que le pendule simple peut être caractérisé dans différents formats qui sont différents du point de vue inférentiel. Ici, le pendule n'est

31. Tout cet exposé est directement emprunté à R. Giere, 1988.

32. Le terme anglais est «template ». Il est utilisé dans le domaine de l'artisanat, au sens de «forme », dans celui de la couture («patron»), en informatique et également en linguistique formelle. Dans ces deux derniers cas, qui sont aussi les deux sens les plus proches de celui que le terme prend dans l'ouvrage de Humphreys, la traduction française habituelle est celle de « gabarit ». C'est ce qui a guidé mon choix de traduction.

33. Paul Humphreys, Extending Ourselves. Computational Science, Empiricism, and Scientific Method, Oxford University Press, 2004. 
pas le representans mais la chose représentée ou, plus précisément, caractérisée. Il est temps de se tourner vers la deuxième branche de la question: comment le pendule lui-même peut-il jouer le rôle de representans?

\subsection{Représentations du système cible}

Prenons un système physique tel qu'une horloge normande, dont le balancier a une masse $m$ et le câble une longueur $l$. Les diverses caractérisations du pendule simple ne s'appliquent pas exactement au mouvement de ce balancier, puisque le câble a une masse, le balancier a un volume et de nombreuses forces s'exercent sur lui, comme la résistance de l'air et les forces de frottement. Par conséquent, afin de représenter ce système au moyen du modèle du pendule simple, on doit en premier lieu écrire les valeurs particulières de $m$ et de $l$ et spécifier les conditions initiales du système : la position et la vitesse du balancier au temps $t_{0}$. Ensuite, on doit déterminer les forces additionnelles qui s'exercent sur le système, à cause du volume du balancier (qui n'est pas une masse ponctuelle), des frottements et de la résistance de l'air. Pourvu que l'on fasse les approximations, distorsions et idéalisations appropriées, on peut finalement écrire une équation qui permet de prédire la position et la vitesse du balancier au temps $t_{i}$. Une telle équation est une spécification de l'équation du pendule simple qui s'applique plus ou moins précisément (selon le but recherché) aux conditions particulières en jeu. Cela revient à ce que Nancy Cartwright $^{34}$ appelle une «description préparée » du système : un système physique, pour qu'un modèle puisse être utilisé pour le représenté, doit luimême être décrit dans les termes utilisés pour décrire le modèle. Notons que, afin d'écrire l'équation, il peut être très utile de faire un dessin schématique du système, afin de spécifier les forces qui s'y appliquent. Un tel schéma luimême consiste en une spécification du schéma du pendule simple qui s'applique - avec distorsions et approximations - au système étudié. De même, afin de déterminer rapidement la période d'oscillation du balancier, il peut être utile de tracer le graphe de son évolution temporelle à partir de l'équation ainsi obtenue.

Dans tous ces cas, quel type d'objet est utilisé pour représenter l'horloge ? Toujours une formule ou une figure particulières : une équation, un schéma, un graphe. Ici encore, afin de tirer des inférences et d'obtenir des informations concernant le comportement du système modélisé, on doit écrire la représentation dans un format particulier, et chaque format a une utilité cognitive particulière, en fonction du but recherché. En aucun cas le pendule n'est en lui-même le representans. Ce dernier ne peut pas être le modèle-I in abstracto, mais plutôt une représentation concrète dans un format particulier, avec laquelle il est possible de conduire un raisonnement.

34. Nancy Cartwright, How the laws of physics lie, Clarendon Press, 1983. 
Autrement dit, les raisonnements se font toujours sur des représentations formatées, et le format utilisé a une influence sur le raisonnement ${ }^{35}$.

Dans quel sens, dès lors, peut-on dire que l'on utilise le modèle du pendule simple pour représenter le comportement de l'horloge ? Ma thèse est qu'un tel propos signifie que l'on utilise des représentations dans les formats qui sont utilisés pour le caractériser. L'équation, le schéma, le graphe représentant le comportement de l'horloge sont dans le même format que, respectivement, l'équation, le schéma et le graphe du pendule simple. Puisque chaque format a sa propre utilité cognitive, le pendule simple peut être utilisé pour tirer différents types d'inférences; ainsi, on ne peut pas identifier le modèle du pendule simple avec un type de représentation unique, au sens défini à la fin du paragraphe 2.2.

Qu'est-ce qui, dès lors, garantit l'unicité du modèle-I ? Pourquoi ne dirait-on pas qu'il y a autant de modèles que de types de représentations ? De nouveau, la boîte à outils goodmanienne est utile. Rappelons brièvement quelques points de l'analyse de Nelson Goodman ${ }^{36}$. Puisqu'une représentation $^{37}$ est une sorte de dénotation, il ne peut pas y avoir de représentation proprement dite d'un objet non existant comme une licorne. En conséquence, ce qu'on appelle communément une «image de licorne » est une image-de-licorne ${ }^{38}$, qui ne dénote rien. Les noms-de-licorne, les descriptions-de-licorne et les images-de-licorne ont en commun leur appartenance à la classe plus large des représentations-de-licorne. Pour représenter un objet existant, par exemple Winston Churchill, on doit utiliser une image, des mots, ou tout autre moyen de représentation. Cette représentation à son tour appartient à une classe de représentations auxquelles une étiquette est appliquée, par exemple «image-de-licorne», « image-de-bouledogue», «nom-d'homme », etc., mais qui ne dénotent pas nécessairement un homme, un bouledogue, ou, a fortiori, une licorne particuliers. Par exemple, une caricature de Churchill qui le dénote sous les traits d'un bouledogue est une représentation de Churchill, puisqu'elle dénote l'homme Churchill, et c'est une représentation-de-bouledogue, sans pour autant dénoter un bouledogue réel. C'est une représentation de

35. On pourrait m'objecter que ma définition du representans comme objet concret particulier est une pétition de principe : puisque le pendule n'est pas un objet concret, il ne peut pas être le representans. Cependant, certaines personnes sont si familières du comportement des pendules qu'elles n'ont même pas besoin d'un crayon et d'un papier pour écrire l'équation et qu'elles peuvent calculer ses solutions sans en tracer une représentation externe. Mais mon propos est que, même quand il est purement imaginé, le pendule ne représente pas en luimême : la représentation qui est imaginée et avec laquelle on raisonne est, elle aussi, dans un format particulier, équation ou graphe, dans la formulation newtonienne ou hamiltonienne.

36. N. Goodman, 1968/1976.

37. N. Goodman parle plutôt de «symbole» que de «représentation », réservant «représentation» au cas des représentations picturales. Ici, j'emploie «représentation » au sens le plus large, correspondant au sens de «symbole » chez Goodman (voir note 1, ch. 1).

38. En anglais, Goodman parle de «unicorn-image». On traduit habituellement, suivant la proposition du traducteur Jacques Morizot, en insérant des tirets. 
Churchill en bouledogue ${ }^{39}$. Revenons-en au pendule simple : l'équation (2) est typiquement ce que Goodman aurait appelé une équation-de-pendule, et plus précisément une équation-newtonienne-de-pendule: elle n'a pas de référent réel, mais elle est présentée sous l'étiquette «équation-de-pendulesimple ». Ma thèse est donc qu'utiliser le pendule simple pour représenter un système cible revient à représenter ce système comme un pendule simple, c'est-à-dire, à proposer une représentation-de-pendule-simple de ce système. Notons d'ailleurs que cela rend assez bien compte de la manière dont la science est enseignée: les étudiants apprennent la loi de l'oscillateur harmonique par le biais de l'exemple du pendule simple. On leur apprend ce qu'est un pendule simple en leur disant que c'est une masse ponctuelle suspendue au bout d'un fil sans masse, en leur présentant l'équation, le graphe, etc., d'une manière semblable à celle dont on enseigne à un enfant ce qu'est une licorne en lui montrant une image-de-licorne ou en lui disant qu'une licorne et un cheval avec une corne. Ainsi, les étudiants apprennent les formats qui sont utilisés en mécanique pour représenter un système comme obéissant à la loi du pendule et se familiarisent avec la manipulation et l'application des représentations dans ces formats à des systèmes physiques. Ils apprennent à représenter des systèmes physiques comme des pendules ${ }^{40}$.

Pour résumer, un modèle-I de la sorte du pendule simple ne peut pas être identifié à un seul et unique type de représentation, mais plutôt à une classe de types de représentations (ici, la classe de toutes les représentationsde-pendule) qui peuvent être logiquement mais non pas inférentiellement équivalents. Un modèle-I ne facilite pas un et un seul type de processus inférentiel; il y a bien plutôt autant de types de raisonnements que de formats différents pour le caractériser.

Une rapide comparaison avec la position de Ronald Giere (2006), avec laquelle la mienne partage plusieurs points, devrait me permettre de rendre mon propos plus clair. Ronald Giere est un défenseur de la conception sémantique des théories selon laquelle les théories peuvent être identifiées à des classes de modèles ; cependant, il insiste sur les aspects cognitifs de la pratique scientifique et à ce titre cherche à donner une définition de la notion de modèle qui s'accommode de la pluralité de ses usages et qui réserve une place importante à l'utilisation des modèles par des agents cognitifs pour représenter des systèmes physiques. C'est ce qui le conduit à définir la relation entre les différents modèles comme une relation de ressemblance -

\footnotetext{
39. En anglais, Goodman parle de «representation as». Jacques Morizot a proposé « représentation en ». Il est des cas, comme dans ce qui suit, où il est plus simple de traduire par « représentation comme». Je prendrai la liberté de le faire.

40. Une telle analyse est très proche de celle que Thomas Kuhn propose, dans les années 1970, lorsqu'il revient sur la notion de paradigme ; les étudiants apprennent ce qu'il appelle des "généralisations symboliques", comme la deuxième loi de Newton $(\mathbf{F}=m \mathbf{a})$, en apprenant à résoudre des problèmes types (exemplars) ; ce faisant, dans le même mouvement, ils apprennent la signification de la loi et ils acquièrent un savoir-faire qui leur permet de décrire les phénomènes du monde comme étant des instances de cette loi.
} 
dont il cherche à donner un sens cognitif - plutôt que d'isomorphisme. A ce titre, Giere insiste sur l'importance de la manipulation concrète de représentations concrètes externes par les scientifiques dans leur raisonnement à propos des phénomènes qu'ils étudient. Au chapitre 5 de son livre, il soulève exactement le même problème que celui que j'ai posé ici : comment un objet abstrait peut-il jouer un rôle dans la représentation, à partir du moment où la représentation consiste en un raisonnement conduit à partir de dispositifs externes ? Il suggère alors que «l'expert utilise les représentations externes dans le but de reconstruire ${ }^{41}$ les aspects du modèle abstrait pertinents pour le problème à résoudre. ${ }^{42}{ }$ Il souligne également la variété des moyens de représentation utilisés pour accéder à un modèle abstrait (dans mes termes, un modèle-I) et le caractériser : "Le modèle physique de l'ADN de Watson et Crick, par exemple, sert aussi à spécifier des caractéristiques d'un modèle abstrait d'ADN, telles que la longueur de l'hélice et les paires de bases permises. » (p. 106) Ainsi, Giere reconnaît que, même dans le cas des modèles abstraits, les scientifiques doivent tracer (concrètement ou mentalement) une représentation dans un format précis. Mais, contrairement à Giere, je ne dirais pas que, ce faisant, ils «reconstruisent» des aspects du modèle abstrait, pour comparer ses propriétés avec les propriétés du système à l'étude. En effet, si la pratique de représentation repose sur des ressemblances, comme Giere le prétend, la ressemblance a lieu entre le modèle physique concret externe de l'ADN et la molécule, et non entre le modèle abstrait et la molécule.

\section{Conclusion}

L'analyse de la fonction représentative des modèles scientifiques était motivée par le projet d'éclairer la faculté des scientifiques à fournir des prédictions et des explications des phénomènes observables. J'ai montré que cette faculté dépend plutôt des formats des représentations particulières que des modèles-I eux-mêmes. En effet, il n'y a pas de relation de représentation authentique entre les modèles-I et les systèmes cibles. Le pouvoir représentatif des modèles-I repose plutôt dans les formats que l'on utilise pour les caractériser et dans les inférences qu'ils nous permettent de tirer. On ne raisonne que sur des représentations formatées.

Ainsi, j'en arrive à conclure qu'il n'y a pas de sémantique spéciale à appliquer aux modèles-I : ils ne représentent pas leur objet en vertu de propriétés qui leur seraient spécifiques en tant qu'entités imaginaires. Même dans le cas de la représentation par le moyen d'un modèle-I, le raisonnement ou la manipulation effectifs sont conduits à partir d'un representans

41. Italiques d'origine.

42. R. Giere, 2006, p. 105. 
particulier, dont le format importe aux prédictions et aux explications qu'on peut en tirer.

Une telle conclusion peut laisser espérer une analyse unifiée de la représentation scientifique par les modèles en général, dans la mesure où la représentation par les modèles-I n'est pas plus mystérieuse que la représentation par le biais de diagrammes, de graphes, d'équations, etc. Cela suggère qu'une étude de la représentation scientifique devrait prendre comme objet d'analyse la variété des formats utilisés dans la pratique scientifique, dans le but de déterminer leurs différentes vertus, la manière dont ils sont en relation les uns avec les autres et avec les principes de la théorie, et le type de raisonnements qu'ils permettent aux scientifiques de mener. 